\title{
PARALISIA DE LARINGE EM CÃO: RELATO DE CASO
}

\author{
LARYNGAL PARALYSIS IN DOG: CASE REPORT
}

\author{
A. M. C. JUNQUEIRA ${ }^{1}$, F. C. BRITTO ${ }^{2}$, B. K. S. ROSA ${ }^{3}$, R. F. CUNHA ${ }^{4}$, \\ M. JESUS ${ }^{5}$, R. Q. STEFANI ${ }^{6}$, F. P. S. MELLO ${ }^{7}$, M. P. FERREIRA ${ }^{8}$
}

\begin{abstract}
RESUMO
A paralisia de laringe consiste na perda da capacidade de abdução das cartilagens aritenóides durante a inspiração. Pode ser congênita ou adquirida, secundária a trauma, neoplasia, polineuropatia ou endocrinopatia. O diagnóstico tem como base os sinais clínicos e exame de imagem da laringe. Uma cadela, de 9 anos de idade, da raça Labrador Retriever foi atendida no hospital veterinário com quadro de dispnéia inspiratória, intolerância ao exercício, sobrepeso, inquietação e taquicardia. Após oxigenoterapia, foram solicitados hemograma, perfil bioquímico, hemogasometria e exame radiográfico do tórax, porém, o diagnóstico definitivo de paralisia de laringe foi obtido por meio da ultrassonografia da região cervical ventral e laringoscopia. Nestes exames foram observados movimentos assimétricos das cartilagens aritenóides durante a inspiração. Desta forma, foi instituído o tratamento emergencial de traqueostomia. Após quatro dias foi realizada a técnica de lateralização aritenóide unilateral. Ao longo de 14 dias de internação, o padrão respiratório da paciente foi restaurado, resultando na alta do animal.
\end{abstract}

PALAVRAS-CHAVE: Laringoscopia. Ultrassonografia. Aritenóides

\section{SUMMARY}

Laryngeal paralysis consists of the loss of the capacity of abduction of arytenoid cartilages during inspiration. The etiology may be congenital or acquired, secondary to trauma, neoplasia, polyneuropathy or endocrinopathy. The diagnosis is based on clinical signs and on the examination of the larynx. A 9-year-old Labrador Retriever was treated at the veterinary hospital, with symptoms of inspiratory dyspnea, exercise intolerance, overweight, restlessness and tachycardia. After oxygen therapy, complete blood count, biochemicals, hemogasometry and chest radiography were requested. The definitive diagnosis of laryngeal paralysis was obtained by ultrasonography of the ventral cervical region and laryngoscopy. Asymmetric movements of the arytenoid cartilages were observed during inspiration. Therefore, emergency tracheostomy treatment was instituted. After the assessment of the operative risks, it was chosen to perform the unilateral laryngeal lateralization procedure. Throughout 14 days of hospitalization, the patient's respiratory pattern was restored, resulting in the discharge of the animal.

KEY-WORDS: Laryngoscopy. Ultrassonography. Arytenoids

\footnotetext{
${ }^{1}$ Mestranda do programa de Pós-Graduação de Ciências Veterinárias. UFRGS. Email:amandamcjunqueira@ gmail.com

${ }^{2}$ Médica veterinária, CRMVRS 14486

${ }^{3}$ Mestranda do programa de Pós-Graduação de Ciências Veterinárias. UFRGS.

${ }_{5}^{4}$ Médica veterinária radiologista e ultrassonografista. PROVET. CRMVSP 43804

${ }^{5}$ Mestranda do programa de Pós-Graduação de Ciências Veterinárias. UFRGS

${ }^{6}$ Médica veterinária especialista em cirurgia. Associação Bichos Gerais. CRMVMG 13267

${ }^{7}$ Médica veterinária. Doutora. Hospital de Clínicas Veterinárias . UFRGS

${ }^{8}$ Docente do departamento de Medicina Animal da Faculdade de Veterinária e Pós-Graduação de Ciências Veterinárias. UFRGS
} 


\section{INTRODUÇ̃̃O}

A paralisia de laringe (PL) consiste na perda da capacidade de abdução das cartilagens aritenóides durante a inspiração (KITSHOFF et al., 2013). Pode ser hereditária, ocorrendo geralmente em animais jovens (KITSHOFF et al., 2013; MILLARD, 2009). Também pode ser adquirida, por traumas, causas iatrogênicas ou em decorrência de neoformações cervicais ou intratorácicas, doenças neuromusculares imunomediadas, endocrinopatias, intoxicação, polimiopatia e polineuropatia idiopática progressiva. Porém, geralmente a causa permanece indeterminada, sendo classificada como idiopática (KITSHOFF et al., 2013; MACPHAIL, 2014). Os sinais clínicos são resultantes do estreitamento da passagem de ar pelas cartilagens aritenóides e os cães podem apresentar angústia respiratória, estridor inspiratório, intolerância ao exercício e disfonia (MACPHAIL, 2014; NELSON; COUTO, 2015).

O diagnóstico definitivo de PL requer exame direto da laringe e a avaliação pode ser realizada com laringoscopia transoral, laringoscopia transnasal, ultrassonografia (US) ou tomografia computadorizada (MACPHAIL, 2014). Contudo, o exame de laringoscopia transoral, com sedação leve, é considerado padrão ouro para o diagnóstico definitivo de PL (BROOME; BURBIDGE; PFEIFFER, 2000; RADLINSKY et al., 2009; SMITH, 2000). A sedação deve ser leve, para que os reflexos laríngeos sejam preservados, visto que alguns agentes ansiolíticos, analgésicos e anestésicos podem reduzir o movimento laríngeo e levar a diagnóstico falso positivo (JACKSON et al., 2004; RADLINSKY et al., 2009). A avaliação da estrutura da laringe e a sua função também podem ser realizadas por meio da US, sem a necessidade de sedação (RUDORF; BARR; LANE, 2001; RUDORF; BROWN, 1998). Além disso, a US pode ser utilizada para diagnosticar presença de neoformações, cistos e colapso laríngeo (RUDORF; BARR; LANE, 2001).

A correção cirúrgica é recomendada para o tratamento dos cães gravemente afetados e diversas técnicas cirúrgicas já foram descritas. Contudo, a lateralização aritenóide unilateral é atualmente considerada como o procedimento padrão ouro para tratar a PL e é frequentemente escolhido pelos cirurgiões (MACPHAIL, 2014; MONNET, 2016). O objetivo deste trabalho é relatar o caso de PL em um cão Labrador Retriever.

\section{RELATO DE CASO}

Foi atendida no Hospital de Clínicas Veterinárias da Universidade Federal do Rio Grande do Sul (HCV-UFRGS), um paciente canino, fêmea, castrada, 9 anos de idade, da raça Labrador Retriever. A tutora relatou que a paciente apresentava grave desconforto respiratório e intolerância ao exercício. Durante o exame clínico observou-se dispnéia inspiratória, sobrepeso, inquietação e aumento da frequência cardíaca. Os demais parâmetros, como temperatura, coloração de mucosas, tempo de preenchimento capilar, hidratação, ausculta cardiopulmonar e exame neurológico encontravam-se dentro da normalidade. Os principais diagnósticos diferenciais, de acordo com o quadro clínico apresentado, foram paralisia de laringe, neoplasia laríngea, laringite obstrutiva, colapso laríngeo, trauma, corpo estranho e massa extraluminal. Foi fornecido oxigenioterapia por sonda nasal e realizados hemograma completo, albumina, alamina aminotransferase (ALT), fosfatase alcalina (FA), colesterol, triglicerídeos, ureia, creatinina, hemogasometria e exame radiográfico do tórax, não sendo observadas alterações nestes exames.

Foi realizado exame ultrassonográfico da região cervical, conforme a técnica descrita por RUDORF; BARR; LANE (2001). Na US foi observado movimento assimétrico das aritenóides (Figura 1), sugestivo de paralisia de laringe bilateral. Posteriormente, foi submetido ao exame de laringoscopia transoral, que foi realizada sob anestesia geral com fentanil $3 \mathrm{mg} / \mathrm{kg}$ e propofol $3 \mathrm{mg} / \mathrm{kg}$, em plano superficial, como já descrito por SMITH, 2000. Durante o exame pôde-se observar perda completa da capacidade de abdução da cartilagem aritneóidea e pregas vocais durante a inspiração (Fiura 2). Após a laringoscopia, foi realizada traqueostomia temporária e internação da paciente, sendo instituído tratamento com prednisona $0,5 \mathrm{mg} / \mathrm{kg}$ por via oral, uma vez ao dia, durante dez dias, enrofloxacina $10 \mathrm{mg} / \mathrm{kg}$ por via oral, durante os doze dias, ranitidina $2 \mathrm{mg} / \mathrm{kg}$ por via subcutânea, duas vezes ao dia, dipirona $25 \mathrm{mg} / \mathrm{kg}$ por via endovenosa, três vezes ao dia e cefalotina $20 \mathrm{mg} / \mathrm{kg}$ /por via endovenosa, três vezes ao dia (durante quinze dias), omeprazol $0,5 \mathrm{mg} / \mathrm{kg}$ por via endovenosa, uma vez ao dia, tramadol $3 \mathrm{mg} / \mathrm{kg}$ por via subcutânea, três vezes ao dia e acetilcisteína $30 \mathrm{mg} / \mathrm{kg}$ via endovenosa, três vezes ao dia (durante dezoito dias), sucralfato 20 $\mathrm{mg} / \mathrm{kg}$ por via oral, três vezes ao dia, durante seis dias e lavagem da sonda de traqueostomia com solução fisiológica. Devido à restrição financeira do proprietário, não foi possível realizar mais exames complementares.

Após seis dias de internação optou-se por realizar procedimento de lateralização unilateral de aritenóide. Como medicação pré-anestésica foi utilizado metadona $0,4 \mathrm{mg} / \mathrm{kg}$ intramuscular, para indução propofol $4 \mathrm{mg} / \mathrm{kg}$ via intravenosa e manutenção com isoflurano, além de bomba de infusão com fentanil $10 \mathrm{mg} / \mathrm{kg}$ e cetamina $1 \mathrm{mg} / \mathrm{kg}$. Após a realização do procedimento cirúrgico e ao longo de doze dias de internação, o padrão respiratório da paciente foi reestabelecido, resultando na alta do animal.

Após um ano, o proprietário relatou que o animal encontrava-se hígido, sem ter apresentado sinais de alteração neste ínterim. 

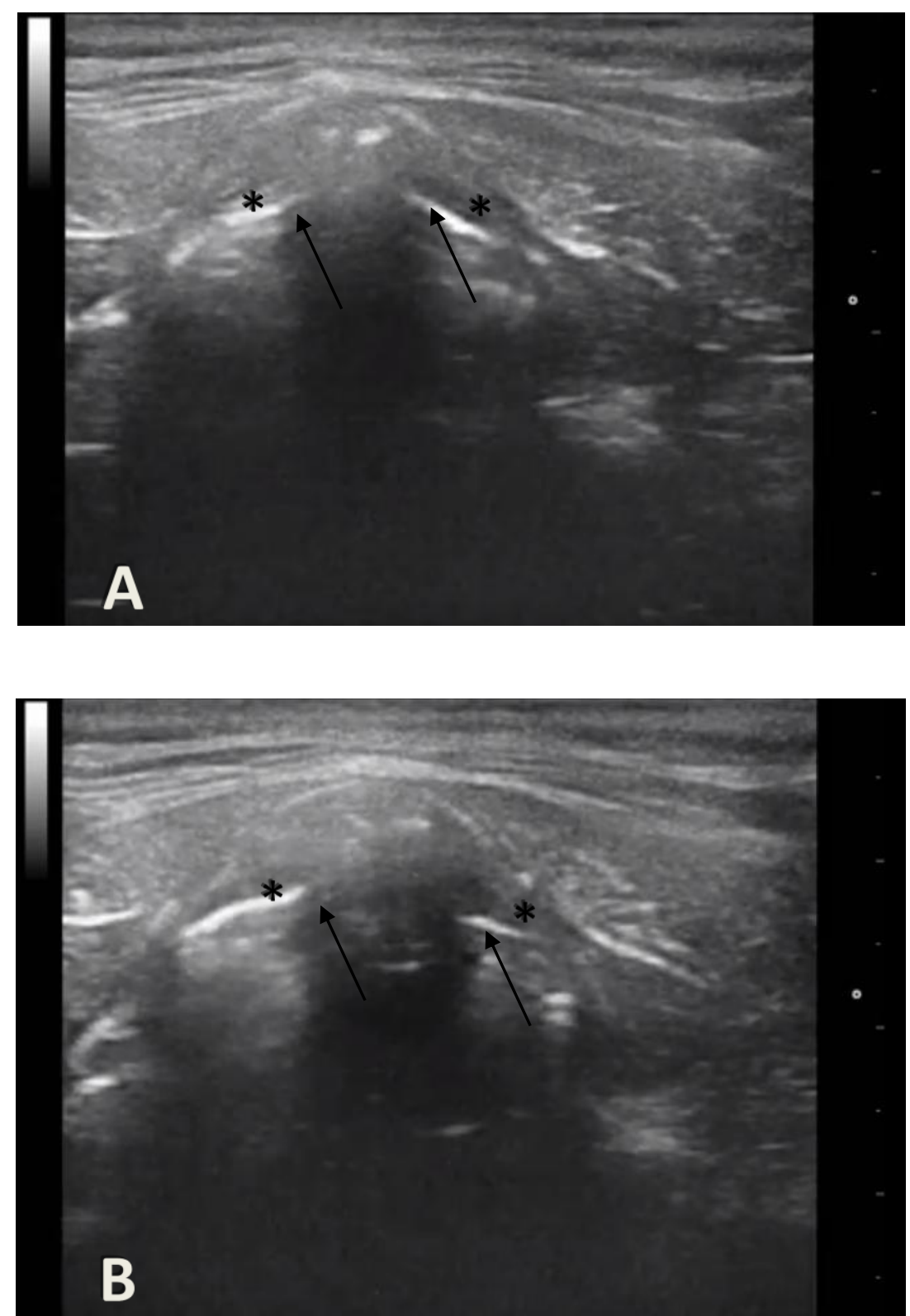

Figura 1 - Imagem ultrassonográfica da região cervical ventral de um cão da raça Labrador Retriever. A e B evidenciando a assimetria (setas) das cartilagens aritenóides (asteriscos), sugestivo de paralisia bilateral.

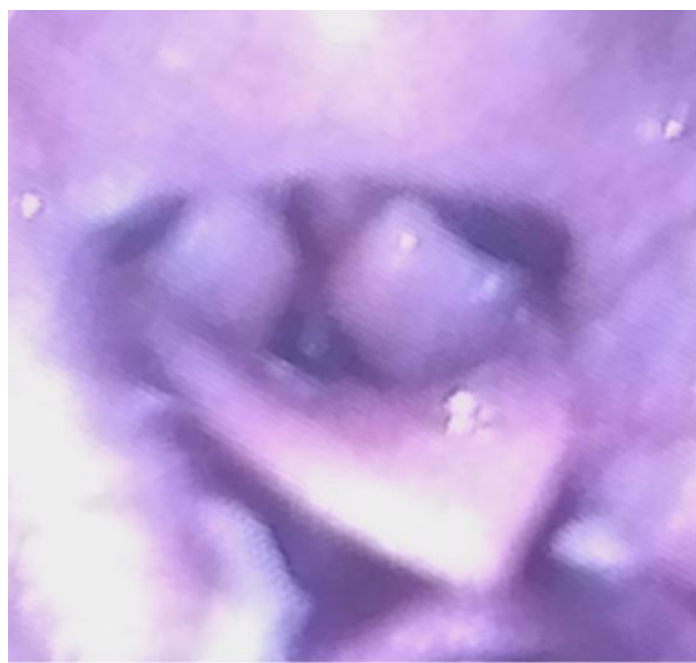

Figura 2 - Imagem da laringoscopia transoral de um cão da raça Labrador Retriever. Durante o exame pôde-se observar a perda completa da capacidade de abdução da cartilagem aritneóidea e pregas vocais durante a inspiração. 


\section{DISCUSSÃO}

A forma adquirida de PL pode ser reflexo de polineuropatia generalizada progressiva, polimiopatia, neoplasia, endocrinopatia, lesão iatrogênica ou idiopática, quando a origem é indeterminada. A PL idiopática, geralmente acomete animais de raças grandes, mais velhos e é bastante descrita em cães da raça Labrador Retriever (KITSHOFF et al., 2013; MACPHAIL, 2014). Perfil semelhante ao da paciente deste relato, que também não apresentava sinais clínicos ou histórico sugestivo de alguma doença que determinasse a causa da PL e, portanto, o caso foi considerado de origem idiopática.

Foi demonstrado em estudo com 11 cães diagnosticados com PL, alterações patológicas nas biópsias em tecido muscular e nervoso da região laríngea e do membro pélvico. Os resultados foram consistentes com desnervação, sugerindo que a PL pode ser sinal precoce de polineuropatia generalizada (THIEMAN et al., 2010). O termo polineuropatia da paralisia de laringe de origem geriátrica (GOLPP) tem sido utilizado para descrever a síndrome de paralisia laríngea idiopática adquirida (MACPHAIL, 2014; STANLEY et al., 2010).

Os sinais clínicos de dispnéia inspiratória, inquietação e intolerância ao exercício são comuns nos pacientes com PL, visto que as cartilagens aritenóides e as pregas vocais se mantém em posição paramediana durante a inspiração, causando obstrução das vias aéreas superiores, conforme foi observado nesse caso (MACPHAIL, 2014). O tratamento inicial deve ser direcionado para melhorar a ventilação e reduzir o estresse do paciente (MACPHAIL; MONNET, 2001) e, dessa forma, foi instituído tratamento emergencial com oxigenioterapia. Foram coletadas amostras sanguíneas para exames complementares, conforme foram citados anteriormente, os quais não apresentaram alterações relevantes, o que é esperado, pois geralmente, hemograma e perfil bioquímico estão normais, a menos que existam doenças concomitantes. Nos pacientes com sinais de polineuropatia generalizada, tais como fraqueza periférica e megaesôfago, recomenda-se mensurar tiroxina livre e concentrações endógenas de hormônio estimulante da tiróide para excluir o hipotireoidismo e os títulos de anticorpos do receptor de acetilcolina para descartar miastenia gravis (MILLARD, 2009). Infelizmente, não foram realizados esses exames devido à restrição financeira do proprietário. A radiografia torácica foi realizada para excluir outras causas de dispnéia e intolerância ao exercício, bem como, para descartar pneumonia por aspiração, edema pulmonar não cardiogênico e megaesôfago (MILLARD, 2009).

O diagnóstico foi obtido com o auxílio da US da região cervical ventral e laringoscopia transoral. Nestes exames observaram-se os movimentos assimétricos das cartilagens aritenóides durante a inspiração. Estudos relataram que quando se compara a US de laringe com a laringoscopia transnasal e a laringoscopia transoral, a sensibilidade e especificidade da US cervical é menor do que a observação direta da laringe e o movimento paradoxal da aritenóide pode gerar resultados falso negativos (RADLINSKY et al., 2009). O movimento paradoxal das aritenóides ocorre por aumento da pressão negativa nas vias aéreas, durante a inspiração e retorna a sua posição por movimento passivo, devido à pressão positiva exercida durante a expiração e é observado em $45 \%$ dos cães com PL (KITSHOFF et al., 2013). Outros fatores que podem afetar a qualidade da imagem estão relacionados com a conformação e temperamento do paciente, bem como a frequência e tipo de transdutor utilizados no exame ultrassonográfico (RUDORF; BARR; LANE, 2001).

A US é útil para descartar lesões em massa nas regiões da faringe e laringe antes da realização da laringoscopia transoral (RADLINSKY et al., 2009). Neste caso, foi importante para direcionar $o$ diagnóstico de paralisia de laringe e ressaltar a necessidade de laringoscopia, exame este que apesar da necessidade de anestesia geral para adequada realização é indicado como padrão-ouro para o diagnóstico dessa patologia (RUDORF; BARR; LANE, 2001).

Segundo RUDORF; BARR; LANE (2001), após diagnóstico de PL confirmado por laringoscopia em 30 cães, observaram ultrassonograficamente assimetria ou ausência de movimento das aritenóides em todos os animais. Destes, em 16 cães, observou-se o movimento ventrodorsal dos processos cuneiformes, agindo como mecanismo compensatório para tensionar as pregas vocais. No caso deste relato foi observado movimento assimétrico das cartilagens aritenóides, o que sugeriu fortemente o diagnóstico de PL.

A paciente permaneceu com dispnéia inspiratória intensa, mesmo após a oxigenioterapia, então optou-se pela traqueostomia de alívio com o intuito de fornecer conforto respiratório. A traqueostomia temporária em cães com PL resulta em prognóstico ruim após a cirurgia de tratamento definitivo da PL, uma vez que os cães que recebem traqueostomia temporária pré-operatória são mais propensos a desenvolver complicações pós operatórias geralmente relacionadas ao desenvolvimento de pneumonia por aspiração (MACPHAIL; MONNET, 2001). Foi realizado procedimento de lateralização unilateral de laringe como tratamento definitivo da PL, sendo este, atualmente, considerado o procedimento de eleição para o tratamento desta doença (KITSHOFF et al., 2013). Diversas técnicas estão descritas na literatura, algumas têm como objetivo ampliar a abertura laríngea com a remoção de uma ou ambas pregas vocais (ventriculocordectomia) ou das cartilagens aritenoideias (laringectomia parcial). Outros procedimentos consistem na lateralização de uma ou das duas cartilagens aritenoideias e outras técnicas objetivam ampliar a laringe ampliando a cartilagem tireoideia (laringofissura) (GRIFFIN IV; KRAHWINKEL, 2005).

Ao longo de doze dias de internação, o padrão respiratório da paciente foi restaurado, resultando na alta do animal. Na ausência de complicações póscirúrgicas, assim como citado na literatura (MACPHAIL, 2014; MILLARD, 2009), o procedimento de lateralização de aritenoide unilateral 
levou a redução do estridor respiratório, aumentou a tolerância ao exercício e o conforto respiratório.

O prognóstico para os pacientes com PL submetidos à cirurgia é considerado de reservado a bom (NELSON; COUTO, 2015). Em estudo realizado com 232 cães com diagnóstico confirmado de PL, que foram submetidos ao procedimento de lateralização unilateral de laringe, a pneumonia por aspiração foi a complicação mais frequente, acometendo cerca de $18,6 \%$ dos pacientes no primeiro ano de cirurgia e $31,8 \%$ durante os primeiros três anos (WILSON; MONNET, 2016). Em estudo realizado com cento e quarenta cães, a média de sobrevivência após a cirurgia foi de um a cinco anos, sendo que 19,3\% dos cães morreram devido a doenças relacionadas com o trato respiratório (MACPHAIL; MONNET, 2001). Neste caso, o animal foi acompanhado por um ano, não apresentando sinal de pneumonia ou qualquer outra complicação.

\section{CONCLUSÃO}

A paralisia de laringe é uma doença comum e é muito importante a adequada avaliação do paciente para excluir doenças concomitantes e possibilitar o tratamento mais adequado para o animal. No paciente deste relato, os exames de imagem foram fundamentais para definir o diagnóstico e planejar o tratamento da paralisia de laringe. Diversas alternativas cirúrgicas estão descritas para o seu tratamento, porém a técnica utilizada, de lateralização aritenóide unilateral, frequentemente apresenta o melhor resultado.

\section{REFERÊNCIAS}

BROOME, C.; BURBIDGE, H. M.; PFEIFFER, D. U. Prevalence of laryngeal paresis in dogs undergoing general anaesthesia. Australian Veterinary Journal, v. 78, n. 11, p. 769-772, 2000.

BURBIDGE, H. M. A review of laryngeal paralysis in dogs. British Veterinary Journal, v. 151, n. 1, p. 7182, 1995.

DAVIES, D. R.; IRWIN, P. J. Degenerative neurological and neuromuscular disease in young rottweilers. Journal of Small Animal Practice, v. 44, n. 9, p. 388-394, 2003.

JACKSON, A. M.; TOBIAS K.; LONG C.; BARTGES J.; HARVEY R. Effects of various anesthetic agents on laryngeal motion during laryngoscopy in normal dogs. Veterinary Surgery, v. 33, n. 2, p. 102-106, 2004.

KITSHOFF, A. M.; GOETHEM, V.B.; STEGEN L.; VANDEKERCKHOY, P.; ROOSTER, H. Laryngeal paralysis in dogs: An update on recent knowledge. Journal of the South African Veterinary Association, v. 84, n. 1, p. 1-9, 2013.

MACPHAIL, C. Laryngeal Disease in Dogs and Cats. Veterinary Clinics of North America- Small Animal Practice, v. 44, n. 1, p. 19-31, 2014.
MACPHAIL, C. M.; MONNET, E. Outcome of and postoperative complications in dogs undergoing surgical treatment of laryngeal paralysis: 140 cases (1985-1998). Journal of the American Veterinary Medical Association, v. 218, n. 12, p. 1949-56, 2001.

MILLARD, P. Laryngeal Paralysis in Dogs. Compendium: Continuing Education for Veterinarians, v.31 n. 5, p. 416-427, 2009.

MONNET, E. Surgical Treatment of Laryngeal Paralysis. Veterinary Clinics of North America Small Animal Practice, v. 46, n. 4, p. 709-717, 2016.

NELSON, R. W.; COUTO, G. C. Distúrbios de laringe e faringe. In: Medicina Interna de Pequenos Animais. 5. ed. Rio de Janeiro: Elsevier, 2015. p. 253.

RADLINSKY, M. G.; WILLIAMS, J.; FRANK, P.M.; COOPER, T.C. Comparison of three clinical techniques for the diagnosis of laryngeal paralysis in dogs. Veterinary Surgery, v. 38, n. 4, p. 434-438, 2009.

RUDORF, H.; BARR, F. J.; LANE, J. G. The role of ultrasound in the assessment of laryngeal paralysis in the dog. Veterinary Radiology and Ultrasound, v. 42, n. 4, p. 338-343, 2001.

RUDORF, H.; BROWN, P. Ultrasonography of laryngeal masses in six cats and one dog. Veterinary Radiology and Ultrasound, v. 39, n. 5, p. 430-434, 1998.

SMITH, M. M. Diagnosing laryngeal paralysis. Journal of American Animal Hospital Association, v. 36 , n. 5 , p. $383,2000$.

STANLEY, B. J.; HAUPTMAN, J.G.; FRITZ, M.C.; ROSENSTEIN, D.S.; KINNS, J. Esophageal dysfunction in dogs with idiopathic laryngeal paralysis: A controlled cohort study. Veterinary Surgery, v. 39, n. 2, p. 139-149, 2010.

THIEMAN, K. M.; KRAHWINKEL, D.J.; SIMS, M.H.; SHELTON G.D. Histopathological confirmation of polyneuropathy in $11 \mathrm{dogs}$ with laryngeal paralysis. Journal of the American Animal Hospital Association, v. 46, n. 3, p. 161-167, 2010.

WILSON, D.; MONNET, E. Risk factors for the development of aspiration pneumonia after unilateral arytenoid lateralization in dogs with laryngeal paralysis: 232 cases (1987-2012). Journal of the American Veterinary Medical Association, v. 248, n. 2, p. 188-194, 2016. 